\title{
Practicality of using a Tether for Electrodynamic Reboost of the International Space Station
}

\author{
Boeing Space \& Communications Group \\ 499 Boeing Boulevard \\ .MC JW.63 \\ P.O. Bor 240002 \\ Huntsville $A L 35802$ \\ (256)461-36+0 John.H.Blumer@Boeing.com
}

John H. Blumer, Benjamin B. Donahue, Michal E. Bangham

\begin{abstract}
ElectroDynamic (ED) Tethers can generate continuous low thrust in a low Earth orbit. An induced current running through the length of the tether reacts with the geomagnetic field to produce thrust. The amount of thrust scales with tether length and current. The International Space Station (ISS) requires periodic reboost to maintain an approximately circular orbit above the Earth. The baseline reboost method is a traditional bi-propellant rocket thruster and tankage system which must to be refueled via Soyuz / Progress or other launch vehicle. The estimated propellant costs associated with keeping ISS in the designated orbit over a 10-year life have been extremely high. The ED Tether would draw energy from the renewable ISS Solar Array electrical power system. Propulsion requirements for ISS vary depending on solar wind and other conditions. It is projected that a ED Tether could provide the majority of the required reboost thrust for ISS for a nominal solar year. For above nominal solar wind years the ISS would have to use the rocket reboost system, but at a greatly reduced level. Thus resulting in substantial cost savings, via the reduction in the number of Earth-to-orbit launch vehicle nighrs to the ISS that must bring reboost propellant. However, the purposes of this paper is to further previous research on an ISS ED Tether and examine the operational and technical issues working against using a ED Tether on ISS. Issues such as Shuttle rendezvous and flight path concerns raise serious safety concems and restrictions on tether use. Tether issues such as tether librations and off angle thrust raise concerns about impacts to microgravity payloads and the long-term effect on ISS orbital path and inclination. Operational issues such as peak power available to an ED Tether and allowable dury cycle may impose severe restrictions on tether design and ultimately limit the practicality of an ED Tether on ISS. Thus while at first glance the cost numbers appear to be strongly in favor of an ED Tether the limitations imposed by safety, operations and technical concerns may severely undermine the economic model. Possible solutions to these problems have been investigated and proposed, however some items like off angle thrust are still being actively investigated for an adequate solution.
\end{abstract}

\section{INTRODUCTION}

While flight experiments have demonstrated the usefulness of tethers in space, to date a long term practical application has not been realized. The intent of this paper is not to design or define a tether design, but to utilize previous research and determine the practicality and restrictions of using an ElectroDynamic (ED) Tether for propulsion on the International Space Station (ISS). The ISS altitude makes it a good candidate for a ED Tether. It has a low Earth obit that provides an efficient use of the Earth magnetic field for ED Tether propulsion. Present implementation for ISS reboost is the use of traditional bi-propellant thrusters. These thrusters require periodic refueling and resupply. The proposed ED tether design is for a supplemental boost system rather than a replacement for the thruster system, due to the design drivers of size and power required for an ED Tether large and powerful enough for ISS Propulsion during worse case years. Also high thrust is required for maneuvers to avoid debris or other objects. The resupply operation for the propellant used by the thrusters has high recurring costs, due primarily to the launch costs of the fuel. Based on launch vehicles used and power available to an ED Tether, cost estimates 
have ranged from a few hundred million to over a billion saved in refueling costs by the use of an ED Tether for supplemental reboost over a 10 year period. A ED Tether reboost system also has several less tangible benefits. A reduction in external contamination via reduction in the use of the ISS hydrazine thrusters has large benefits for science payloads and the power producing solar arrays. Another benefit is an ED Tether thrust is approximately equal to ISS drag, thus a high percentage of microgravity payloads should be able to operate for long periods of time as compared to having to halt operation when the higher thrust hydrazine thruster are activated.

Previous work and papers such as The Tethers In Space Handbook (Smithsonian Astrophysical Observatory, 1997). Electrodynamic Tethers For Spacecraft Propulsion (Johnson, 1998), and Space Station Reboost with Electrodynamic Tethers (Vas, 2000) have looked at using a ED Tether for propulsion and proposed possible designs. For this paper a new tether design is not proposed, instead a reference design is used to determine restrictions and design issues that might prevent the use of a ED Tether on ISS. The referenced ED Tether design and cost estimates utilized by this paper are described in detail in the Space Station Reboost with Electrodynamic Tethers (Vas, 2000). The ED Tether design baseline consists of a $7 \mathrm{kM}$ long tether. Both 5 and $10 \mathrm{~kW}$ ED Tethers are considered. The design is a conventional tether with $5 \mathrm{~km}$ of the tether insulated and the bottom $2-\mathrm{km}$ end of the tether left uninsulated to collect electrons. Using numbers from the $V$ as (2000) paper it has been estimated a $7.55 \mathrm{~kW}$ tether is needed to equalize the nominal aerodrag of ISS over an 11 year solar cycle, assuming a $100 \%$ duty cycle and disregarding off angle thrust issues. Figure 1 indicates the original baseline design with a Nadir pointing tether using the ISS plasma contactor.
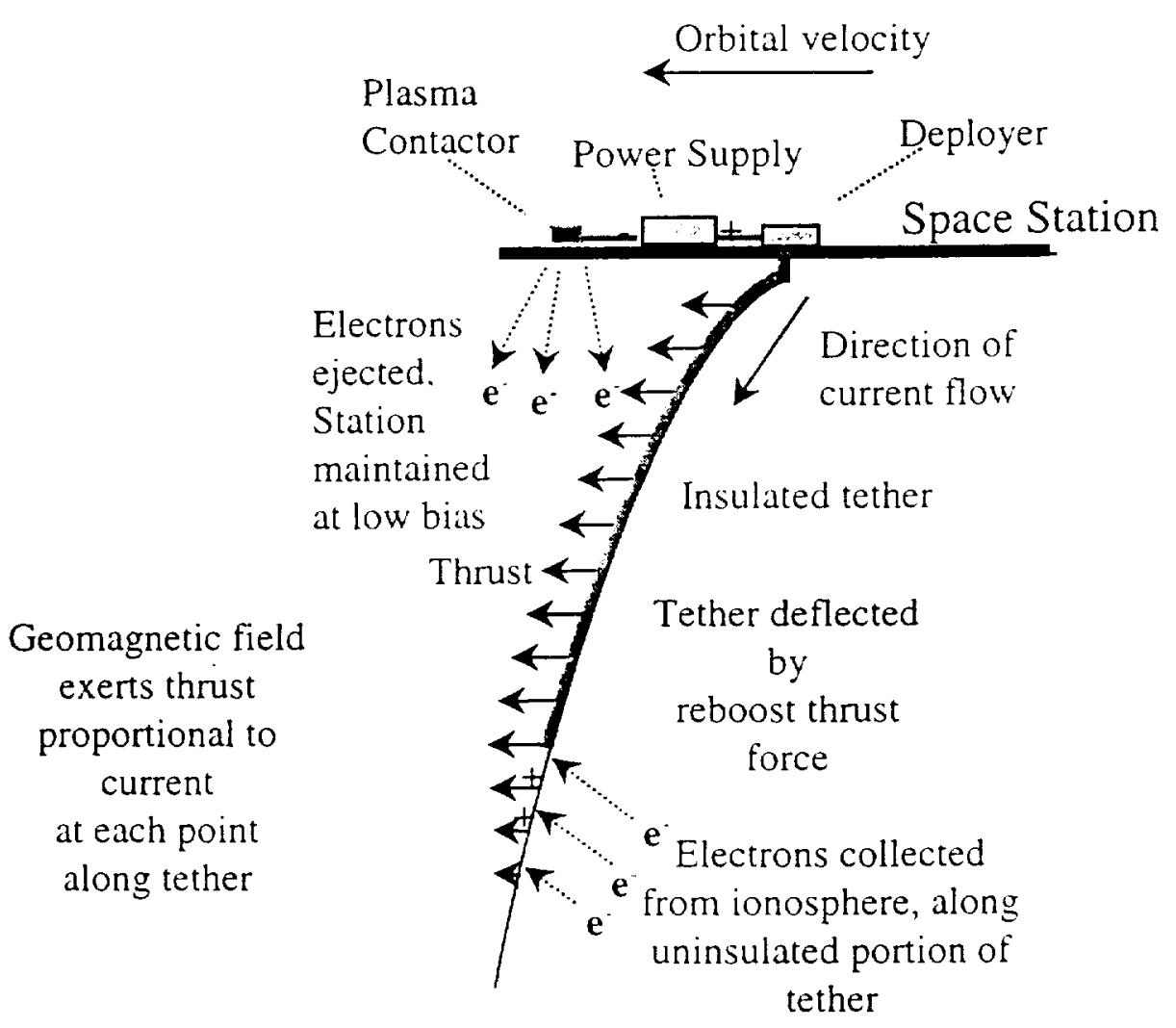

FIGURE 1. ISS ED Tether Design Proposed by Space Station Reboost with Electrodynamic Tethers (Vas, 2000) 


\section{ISS DESIGN DRIVERS}

On a manned platform such as ISS, safety and operational constraints are major design drivers. Using the previously described ED Tether, the ISS interface issues were investigated for not only for technical design problems, but ISS operations concerns, such as vehicle tlight operations and crew safety.

\section{ISS Plasma Contactor}

Preliminary design was a 7-KM long ED Tether hanging down from ISS pointing toward Earth (Nadir pointing). The ISS Plasma contactor was investigated to determine if it could support the additional requirements of a $5-10 \mathrm{~kW}$ tether. The ISS Plasma contactor will support up to 10 amps, of which it is estimated that ISS will need 2-3 amps for normal operations. There is also a back-up unit, but its use would not be permitted for normal usage by a ED Tether. Thus it appears the ISS plasma contactor could support a $5 \mathrm{~kW}$ ED Tether, but questions remain if the loss of design margin in the plasma contactor is acceptable to the ISS design team. A $10 \mathrm{~kW}$ Tether would require approximately the entire design margin of the ISS plasma contactor and thus a supplemental plasma contactor would be required.

\section{ISS Attachment Locations and Shuttle Operations}

The most obvious attachment points in regards to easiest interface are the payload pallet locations on the two outboard truss sections. These have readily available attachment points as well as power and data ports. However, the power ports are limited to $3 \mathrm{~kW}$ peak. Also the points are far from the C.G. (Center of Gravity) for ISS. One possible design solution is to use a ED Tether at each end of the truss. However, the additional cost of two tethers, reels, etc and the question of getting more than $3 \mathrm{~kW}$ (even with two ports combined) caused concern for this option. One possible benefit is that the positions are far enough off of the shuttle tlight path so that they would not have to be reeled in during rendezvous. However, initial feedback from ISS safety was that even for the outboard truss Nadir position, the tether is to close for comfort and may have to be reeled in. One possibility for the first couple of years the ED Tether is deployed on ISS, the tether would have to be reeled in; but, over time as the tether motion is better defined, ISS Safety may allow the tether to stay deployed during shuttle rendezvous operations. Subsequent research indicated the most desirable attachment points would be near the center of gravity for ISS to minimize other design issues, such as having ISS CMGs (Control Mass Gyros) compensate for off-axis thrust. The ideal position, near the ISS center of gravity and with viable structural attachment points and power connections, is the $\mathrm{Zl}$ truss section. However, that would place the tether in the Space Shutte path used for rendezvous with ISS. Several options were investigated to resolve the problem. The use of a reel to retract the tether prior to each Space Shuttle rendezvous was the initial candidate. For the purpose of this study, a reel design such as the one utilized for the Space Shuttle Tethered Satellite System tlights was utilized. Although, for actual use a revised design more optimized to the ISS ED Tether size would be used. Shuttle rendezvous operations are estimated at 5 or 6 times per year after ISS assembly is complete. Thus, use of the reel for 50-60 operations over a 10-year life and its estimated reliability and the associated degradation on the tether was a significant concern. Due to safety requirements a redundant or backup system is required. Also. other concerns like minimum time to retract a tether in case of an emergency drove the backup system design. A tether cutter or guillotine was selected as the backup design. However, the time for the tether to dritt away from ISS is also a concern. In an emergency situation where the CRV (Crew Rescue Vehicle) is used, the time to remove the techer from the CRV flight path might be as little as two minutes. Thus possible use of thrusters on the end mass to accelerate the tether away from ISS is being investigated

A space pointing or Zenith implementation is also being investigated, with attachment to the Z1 truss. A Zenith design would require the plasma contactor or other electron-emitting device, such as a Field Emitter Array Cathode, to be on the end mass end of the tether. Other design issues, such as providing power to the plasma contactor on the end mass would make the Zenith orientation a more complex design. However, it would minimize the safety and operational concerns of a tether being in the path of the Space Shuttle, since the Space Shuttle docks from the bottum or Nadir side of ISS. A reel and a backup cutter would still be required, but the number of reeling operations, as well as most of the safety concerns during the 10-year design life. would be greatly reduced. 


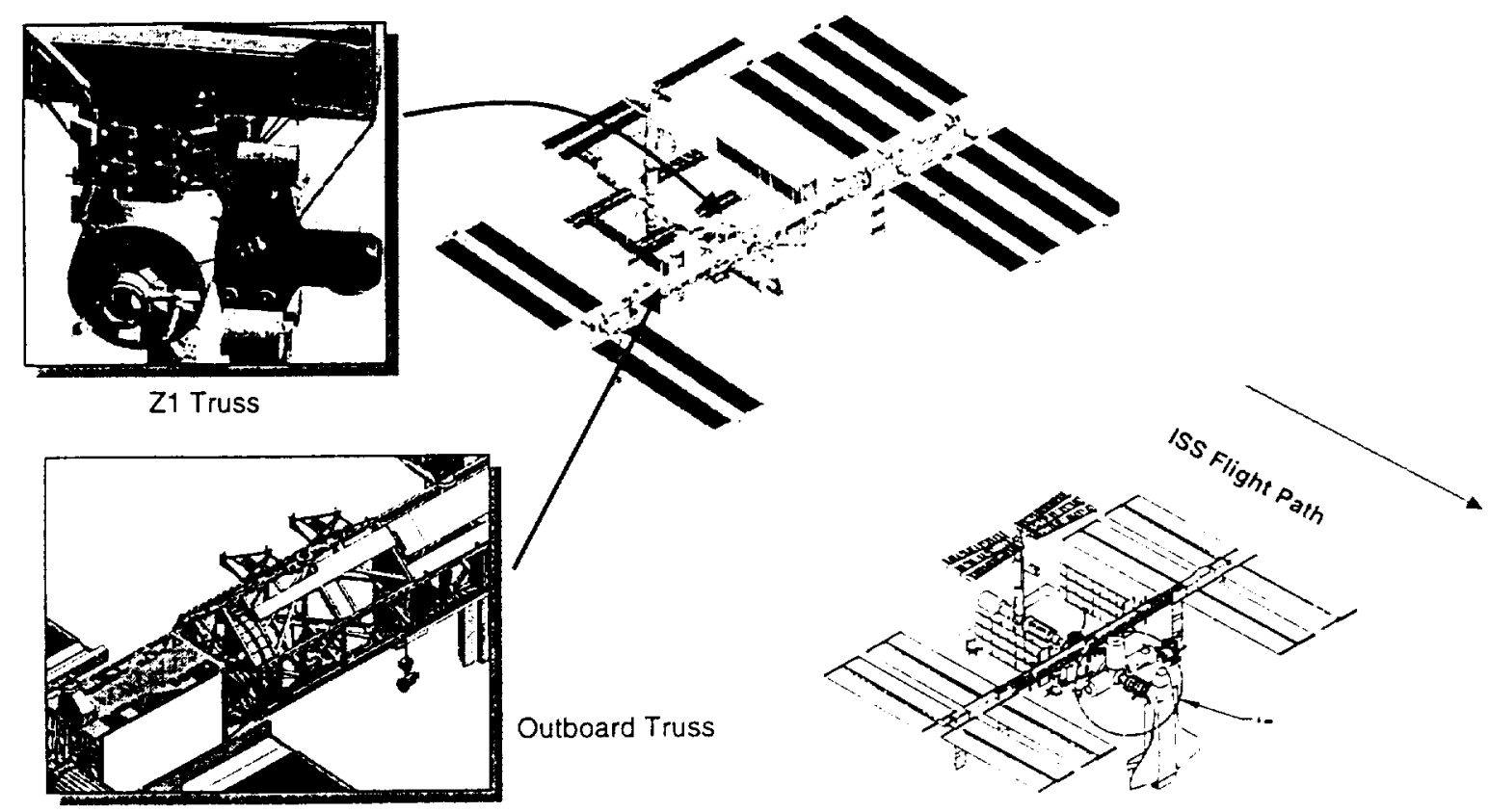

FIGURE 2. Possible ISS Attachment Locations and Nominal Shuttle Docking Position.

Power

The most readily available power ports for external connections, which are located on the outboard truss, are limited to $3 \mathrm{~kW}$ peak. Power ports used during construction of ISS, but not used after ISS assembly is complete, are the prime candidates for $6 \mathrm{~kW}$ power. While it might be possible to use multiple ports for more than $6 \mathrm{~kW}$ of power, the practical limitations of such a design and availability of ISS power discourage a multiple power port implementation. Other candidates are available, such as the connections to ISS truss power distribution equipment; but without a designed-in power port, their accessibility is more difficult and limited. Subtracting DC/DC converter losses for conversion of ISS 120/160 VDC power to the ED Tether voltage of up to 2000 volts DC. plus other avionics, would limit power available to the tether. from a $6 \mathrm{~kW}$ source to slightly more than $5 \mathrm{~kW}$. Another concern is available ISS power. Due to projected ISS power usage, available power for a ED Tether or any external device is very limited. A ED Tether may be limited to a duty cycle of $25-50 \%$ or less due to available power, as well as other operational constraints. (E.g. operational only during periods of surplus power and/or when the solar arrays are illuminated by the Sun and thus when the ISS power system is the most efficient).

\section{EMI, EVA, Charged Plasma, \& Tether Angle}

Several other issues were design drivers or concerns. EMI from the DC/DC converters and other avionics are subjected to the strict ISS EMI requirements. However, at this time this issue should not require other than good design practices to overcome.

There was concern over Astronaut EVA (Extra Vehicular Activity) or space walks near the tether. Analysis shows the electrons flowing through the local plasma, due to the ED techer. have very little energy and thus little penetration capability. The electrons would be stopped by the surface of the EVA suit. However, the present plan is to turn off the tether at any time the astronauts are in the vicinity of the tether to guarantee their safety. Another plasma concern is the interaction with ISS and its plasma contactor, if the ED Tether design requires an additional electron emitter. This analysis is still in work, but preliminary estimates indicate no significant issues. 
A bigger design driver is the cone angle of the Tether during operation. The more desirable locations are near the center of gravity for ISS, but there are several nearby protrusions, such as the Russian Solar arrays on the Science Power Platform (Zenith orientation), ISS modules, ISS thermal radiators, etc. Using worse case cone angle scenarios, the tether could possibly impact ISS structure. Proposed baseline design is to use a rigid structure or deployable boom to extend tether away from ISS, so that assuming the worse case angle it would not impact any ISS structure or vehicle. Since tether location and tether design are not complete, the worse case cone angle and thus boom length are still in work.

\section{Microgravity Impacts}

Another issue heavily impacted by the ED Tether attachment location is the microgravity environment on ISS. Depending on location when in the thrust mode. the ED Tether can shift the center of mass and thus reposition the microgravity contours on ISS. Thus the ED Tether can move the microgravity contour such that one module in ISS has an improved microgravity environment while another has a degraded microgravity environment. One positive impact as compared to a bi-propellant thruster is that the tether thrust is approximately equal to the ISS aero drag. Thus all the micro gravity sensitive payloads that have to be shut down during thruster operation should be able to operate while the ED Tether is in operation, per feedback from the microgravity community. However, the microgravity disturbances from Libration and off angle thrust issues have not yet been adequately characterized. So until these issues are adequately resolved the issue of microgravity impact to payloads remains open.

\section{Libration and Off Angle Thrust}

As the orbit precesses, the travel of ISS is not always due east in regards to the face of the earth. ISS with an inclination of 51.6 degrees has only a small percentage of its orbital time actually traveling eastward. The ED Tether interaction with the Earth's magnetic field produces an eastward thrust (note: some variation due to local disturbances and the geomagnetic field not being perfectly aligned with the poles does occur). Thus an out of plane thrust is produced. A worse case example is crossing the equator with a 51.6-degree inclination orbit. The induced thrust along the orbit is only $62 \%$ of the total thrust magnitude, while the out of plane component is at $78 \%$ of the total thrust magnitude. Out of plane forces can resonate with out of plane motion, thus driving techer motions to large amplitudes. This is a significant factor driving tether and boom design. Thus boom length will be a function of the height and proximity of possible impact targets, such as the Russian Science Power Platform and the worse case tether amplitude or angle. A second issue related to tether amplitude is the disturbance to the microgravity environment of ISS and how the disturbances effect the microgravity sensitive payloads. A third issue is inclination change due to off angle thrust. The concern is that continued out of plane thrust will eventually cause an orbital inclination change, slowly pushing the ISS inclination lower, toward the equator. Two different model runs were performed. Neither model took into account variations in the magnetic field or tether librations. The two models used different software programs and had slightly different results. The second model with the higher indicated inclination change modeled both .43 and .7 Newtons of thrust, with a variable duty cycle. Using .43 Newtons, the second model indicated an inclination change in degrees of $.07382, .28296$, and .966653 over 10 years, for a duty cycle of $25 \%, 50 \%$, and $100 \%$, respectively. Using .0 .7 Newtons, the second model indicated an inclination change in degrees of $.12017, .46063$, and .1 .57341 over 10 years, for a duty cycle of $25 \%, 50 \%$, and $100 \%$, respectively.

One obvious workaround around for the out of plane thrust issue, is to limit thrust during periods of time when the ISS orbit is mostly eastward, however this solution could limit the ED Tether thrust duty cycle, depending on thrust level and allowable ISS inclination change and Tether Libration. One obvious solution, for the inclination change issue, is to use the ISS bi-propellant system to thrust slightly off-angle when used; thus, countering the effect of the ED Tether inclination change. However, one down side is a slight increase in the usage of bi-propellant fuel. Several more complex solutions that have been identified and will be addressed in future studies. These solutions include the use of a controllable end mass and nodes along the length of the tether. The nodes would control current through the length of the particular section of tether; this solution has promise in particular for the Libration and tether oscillation concern. Another possible solution related to inclination change is to use the tether for propulsion for the majority of the orbit. Than switch over to power generation mode prior to crossing the equator, which is the part of the orbit with the highest angle and thus produces the most significant change in orbit inclination. The short period 
of out of plane drag or power mode, while crossing the equator, should balance out the inclination change caused by the longer duration thrust mode. A similar concept is to use the ED Tether for power generation over several orbits, during peak ISS power demand, in addition to the normal thrust mode. This concept has the advantage of fewer disturbances to the micro-gravity sensitive payloads. Thus the ratio of propulsion vs. drag or power mode is high, with the added benefit of producing electrical power for ISS. However, additional costs for a dual mode tether and further issues on microgravity disturbances and libration complicate this design concept. A detailed simulation model of the off angle thrust and Libration is planned for the next study phase and should yield accurate numbers to help select the appropriate design solution.

\section{Self-sufficient Zenith Design}

Due to operational constraints and the amount of tether reeling required for a Nadir pointing tether, the more complex Zenith design with a plasma contactor or electron emitter on the end mass was chosen as the baseline approach. The Zenith design also affords a better opportunity for other options. Since power availability from ISS is limited to $6 \mathrm{~kW}$ peak and with a very restricted duty cycle, the possibility of a self-sufficient ED Tether was investigated. A design with a micro-satellite type structure as the end mass with large solar arrays is a possible concept. The solar array would provide power for the ED Tether propulsion as well as avionics, reaction wheels, thrusters, etc. within the MicroSat. Using high efficiency solar cells for a small array size and when combined with the distance from ISS (E.g. $7 \mathrm{~km}$ ) the issue of shadowing ISS solar arrays is minimized. The $7-\mathrm{km}$ distance of the avionics and power converter from ISS also helps with other ISS design drivers such as EMI and arcing. Power for the seldom used reel and tether cutter, which is still attached to ISS, would still be from ISS. This design is indicated in Figure 3.

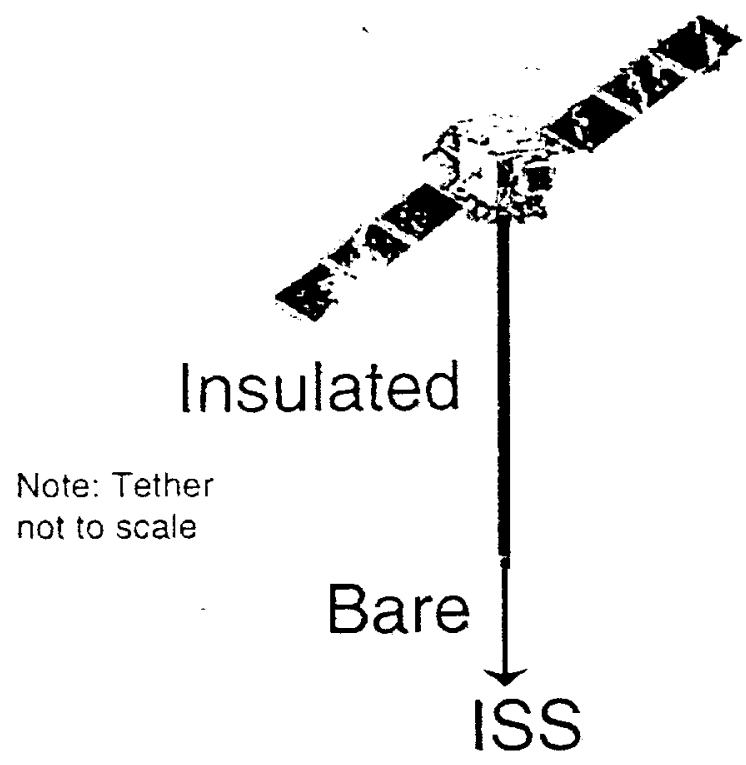

\section{Solar Array $=6.6-15 \mathrm{KW}$}

Day and night operation

Includes tether endmass

avionics - reaction wheels, plasma contactor, PMAD, controller, cold gas jets, sun/earth sensor, array gimbals, IMU, etc.

Possible use of surplus solar array power for ISS

FIGURE 3. Preliminary ISS ED Tether Design.

\section{CONCLUSION}

The main technical issues that limit use of a ED Tether on ISS are libration and the possible issue of inclination change due to off angle thrust. Microgravity disturbances also need to be quantified prior to utilizing a tether for propulsion on ISS. ISS Power and operational issues also limit the ED duty cycle. With ISS power availability at it planned levels, even if the off angle thrust issue is resolved, the tether duty cycle could still be limited to a maximum duty cycle of $50 \%$, or less. The safety and operational usage of a tether is further limited by Shuttle and CRV operations. A ED Tether in a Zenith orientation greatly reduces the impact of a Space Shuttle rendezvous with ISS 
and a CRV launch. A disadvantage of the Zenith orientation is increased complexily and the issue of providing power to the tether and plasma contactor. The option of using a MicroSat as the end mass, with large solar panels, resolves the issue of providing power and the availability power from ISS. However, that option with a larger and probably heavier end mass could further complicate libration and microgravity concerns. The main goal of this study was to identify the barriers to using a ED Tether on ISS and, if possible, identify design solutions. The remaining barriers, such as off angle thrust and Libration, which require a detailed simulation model to resolve, will be tackled in phase 2 of this study.

\section{ACKNOWLEDGMENTS}

I wish to acknowledge Marshall Space Flight Center (MSFC) and Les Johnson for their support through the ISS Reboost Using Electrodynamic Tether Integration Study contract.

\section{REFERENCES}

Cosmo, M.L., and Lorenzini, E.C., Tethers In Space Handbook, The Smithsonian Astrophysical Observatory, Cambridge, 1997. Johnson, Les; Estes Robert D.; Lorenzini, Enrico; Martinez-Sanchez, Manuel; Sanmartin, Juan; and Vas, Irwin, "Electrodynamic Tethers For Spacecraft Propulsion", American Institute of Aeronautics and Astronautics (AlAA), AIAA 98-0983. Nevada (1998).

Vas. Irwin E.; Kelly. Thomas J.: and Scarl. Ethan A.. "Space Station Reboost with Electrodynamic Tethers," Journal of Spacecraft and Rockets (A/AA), Volume 37. Number 2. Virgina (2000) 